\title{
A characterization of supersolubility of finite groups
}

Jinbao Li*

${ }^{\text {"Correspondence: }}$

leejinbao25@163.com

Department of Mathematics, Chongqing University of Arts and Sciences, Chongqing, 402160, P.R. China

\begin{abstract}
In this paper, we study the structure of finite groups whose minimal subgroups are $\lambda$-supplemented and give a new characterization of supersolubility of finite groups. MSC: 20D10; 20D15; 20D20

Keywords: finite groups; minimal subgroups; $\lambda$-supplemented subgroups; supersoluble groups
\end{abstract}

\section{Introduction}

All groups considered in this paper are finite.

It is an interesting topic in group theory to study the generalized permutable subgroups and generalized supplemented subgroups. The present paper is a contribution to this line of research.

Let $G$ be a group and $H$ be a subgroup of $G$. $H$ is said to be a permutable (or quasinormal) subgroup of $G$ if $H$ permutes with every subgroup of $G$. If $H$ permutes with every Sylow subgroup of $G$, then we call $H$ an $S$-permutable (or $S$-quasinormal) subgroup of $G$. Recently, these concepts have been generalized by many authors. In [1], Ballester and Pedraza-Aguilera called $H$ an $S$-quasinormally embedded subgroup of $G$ if a Sylow $p$-subgroup of $H$ is also a Sylow $p$-subgroup of some $S$-quasinormal subgroup of $G$ for each prime $p$ dividing the order of $H$. In [2], Skiba introduced the concepts of weakly $S$-permutable subgroups and weakly $S$-supplemented subgroups. $H$ is said to be weakly $S$-supplemented (weakly $S$-permutable) in $G$ if $G$ has a subgroup (subnormal subgroup) $T$ such that $G=H T$ and $H \cap T \leq H_{s G}$, where $H_{s G}$ denotes the subgroup of $H$ generated by all those subgroups of $H$ which are $S$-quasinormal in $G$. As a generalization and unification of the above two different kinds of embedding property of subgroups, Li and Chen in [3] introduced the concept of $\lambda$-supplemented subgroups. Let $H$ be a subgroup of a group $G$. $H$ is said to be $\lambda$-supplemented in $G$ if $G$ has a subgroup $T$ such that $G=H T$ and $H \cap T \leq H_{S E}$. In this definition, $H_{S E}$ denotes the subgroup of $H$ generated by all those subgroups of $H$ which are $S$-quasinormally embedded in $G$. Using this idea, some new characterizations of $p$-supersolubility of finite groups are obtained in [3] under the assumption that the maximal subgroups of the Sylow subgroups of some special subgroups are $\lambda$-supplemented.

In this paper, we study the structure of groups with some $\lambda$-supplemented minimal subgroups and give a new characterization of supersolubility of finite groups. In fact, the main result established in this paper provides a new criterion, in terms of the $\lambda$-supplemented

(c) 2012 Li; licensee Springer. This is an Open Access article distributed under the terms of the Creative Commons Attribution License (http://creativecommons.org/licenses/by/2.0), which permits unrestricted use, distribution, and reproduction in any medium, provided the original work is properly cited. 
cyclic subgroups of order prime or 4 contained in the generalized Fitting subgroups, for a group to be contained in a saturated formation containing all supersoluble groups.

We prove the main result in Section 3. Section 4 includes some applications of our main result, which unify and extend many previous known results; and the paper concludes with a partial answer to a question of Skiba.

The notation and terminology in this paper are standard. The reader is referred to [4] if necessary.

\section{Preliminaries}

We cite here some known results which are useful in the sequel.

Lemma 2.1 [3, Lemma 2.3] Let $G$ be a group and $H \leq K \leq G$.

(1) Suppose that $H$ is normal in $G$. Then $K / H$ is $\lambda$-supplemented in $G / H$ if and only if $K$ is $\lambda$-supplemented in $G$.

(2) If $H$ is $\lambda$-supplemented in $G$, then $H$ is $\lambda$-supplemented in $K$.

(3) Suppose that $H$ is normal in $G$. Then $N H / H$ is $\lambda$-supplemented in $G / H$ for every $\lambda$-supplemented subgroup $N$ of $G$ satisfying $(|N|,|H|)=1$.

Lemma 2.2 [3, Lemma 2.2] Suppose that $U$ is $S$-quasinormally embedded in a group $G$. If $U \leq O_{p}(G)$, then $U$ is $S$-quasinormal in $G$.

Lemma 2.3 [5, Lemma 2.8] Let $H$ be a normal subgroup of a group $G$. Then $H \leq Z_{\infty}^{\mathcal{U}}(G)$ if and only if $H / \Phi(H) \leq Z_{\infty}^{\mathcal{U}}(G)$.

Lemma 2.4 [6, Theorem 3.1] Let $p$ be the smallest prime dividing the order of a group $G$ and $P$ be a Sylow p-subgroup of $G$. Then $G$ is p-nilpotent if and only if every cyclic subgroup $H$ of $P$ of order $p$ or order 4 (if $P$ is a non-Abelian 2-group and $H \nsubseteq Z_{\infty}(G)$ ) is $\lambda$ supplemented in $G$ provided that $H$ does not possess any supersoluble supplement in $G$.

Lemma 2.5 [6, Theorem 3.2] Let G be a group with a normal subgroup E such that G/E is supersoluble. Suppose that for every non-cyclic Sylow subgroup P of E, every cyclic subgroup $H$ of $P$ of prime order or order 4 (if $P$ is a non-Abelian 2-group and $H \nsubseteq Z_{\infty}(G)$ ) is $\lambda$ supplemented in $G$ provided that $H$ does not possess any supersoluble supplement in $G$. Then $G$ is supersoluble.

Lemma 2.6 [7, Chapter X] Let $\mathrm{G}$ be a group. Then

(1) If $G$ is quasinilpotent and $N$ is a normal subgroup of $G$, then $N$ and $G / N$ are quasinilpotent.

(2) If $N$ is a normal subgroup of $G$, then $F^{*}(N)=N \cap F^{*}(G)$.

(3) $F(G) \leq F^{*}(G)=F^{*}\left(F^{*}(G)\right)$. Moreover, if $F^{*}(G)$ is soluble, then $F^{*}(G)=F(G)$.

\section{Main results}

Lemma 3.1 Let $N$ be a non-trivial normal p-subgroup of a group $G$ of exponent $p$. Suppose that every minimal subgroup of $N$ without any supersoluble supplement in $G$ is $\lambda$ supplemented in G. Then $N \leq Z_{\infty}^{\mathcal{U}}(G)$.

Proof Assume that $\Phi(N) \neq 1$. We check that $N / \Phi(N)$ satisfies the hypothesis. Let $H / \Phi(N)$ be a minimal subgroup of $N / \Phi(N)$. Then $H / \Phi(N)=\langle x\rangle \Phi(N) / \Phi(N)$, where $x \in H \backslash \Phi(N)$. 
By the hypothesis, $x$ is of order $p$. If $\langle x\rangle$ has a supersoluble supplement $T$ in $G$, then $T \Phi(N) / \Phi(N)$ is a supersoluble supplement of $H / \Phi(N)$ in $G / \Phi(N)$. Suppose that $\langle x\rangle$ does not have a supersoluble supplement in $G$. Then $\langle x\rangle$ is $\lambda$-supplemented in $G$ by the hypothesis and so $G$ has a subgroup $T$ such that $G=\langle x\rangle T$ and $\langle x\rangle \cap T \leq\langle x\rangle_{S E}$. If $\langle x\rangle_{S E}=1$, then $\langle x\rangle \Phi(N) / \Phi(N) \cap T \Phi(N / \Phi(N))=1$. If $\langle x\rangle_{S E} \neq 1$, then $\langle x\rangle$ is $S$-quasinormal in $G$ by Lemma 2.2 and therefore $H / \Phi(N)$ is $S$-quasinormal in $G / \Phi(N)$. Thus, we have that $H / \Phi(N)$ is $\lambda$-supplemented in $G / \Phi(N)$. Hence, $N / \Phi(N)$ satisfies the hypothesis and consequently $N / \Phi(N) \leq Z_{\infty}^{\mathcal{U}}(G / \Phi(N))$ by induction. By Lemma $2.3, N \leq Z_{\infty}^{\mathcal{U}}(G)$. Now we consider the case $\Phi(N)=1$. Let $H / K$ be any chief factor of $G$ with $H \leq N$ and $L / K$ be a minimal subgroup of $H / K$ which is normal in some Sylow $p$-subgroup of $G / K$. Then $L=\langle x\rangle K$ for some $x \in L \backslash K$. Suppose that $\langle x\rangle$ has a supersoluble supplement $T$ in $G$. Then $\langle x\rangle \leq T$ or $\langle x\rangle \cap T=1$. If $\langle x\rangle \leq T$, then $H / K$ is of order $p$ since $G / K=T / K$ is a supersoluble group. Assume that $\langle x\rangle \cap T=1$. Then $N \cap T$ is normal in $G$ and $N /(N \cap T)$ is of order $p$. By Lemma 2.1, $N \cap T$ satisfies the hypothesis and so $N \cap T \leq Z_{\infty}^{\mathcal{U}}(G)$ by induction. It follows that $N \leq Z_{\infty}^{\mathcal{U}}(G)$ since $N /(N \cap T)$ is of order $p$. If $\langle x\rangle$ does not have a supersoluble supplement in $G$, then by the hypothesis, $G$ has a subgroup $T$ such that $G=\langle x\rangle T$ and $\langle x\rangle \cap T \leq\langle x\rangle_{S E}$. If $\langle x\rangle \cap T=1$, then $N \leq Z_{\infty}^{\mathcal{U}}(G)$ as above. If $\langle x\rangle_{S E} \neq 1$, then $\langle x\rangle$ is $S$ quasinormal in $G$ by Lemma 2.2. Hence, $O^{p}(G) \subseteq N_{G}(\langle x\rangle)$. Since $L / K=\langle x\rangle K / K$ is also a normal subgroup of some Sylow $p$-subgroup of $G / K$, we obtain that $L / K$ is normal in $G / K$ and so $H / K$ is of order $p$, which implies that $N \leq Z_{\infty}^{\mathcal{U}}(G)$. Thus, the proof is complete.

Theorem 3.2 A group $G$ is supersoluble if and only if $G$ has a normal subgroup $E$ such that $G / E$ is supersoluble and for every non-cyclic Sylow subgroup $P$ of $F^{* \prime}(E)$, every cyclic subgroup $H$ of $P$ of prime order or order 4 (if $P$ is a non-Abelian 2-group and $H \nsubseteq Z_{\infty}(G)$ ) is $\lambda$-supplemented in $G$ provided that $H$ does not possess any supersoluble supplement in $G$.

Proof The necessity is obvious and we consider only the sufficiency. Suppose it is false and let $G$ be a counterexample with $|G|+|E|$ minimal. Then $G$ has the following properties.

(1) $F=F^{\prime \prime}(E)=F(E) \neq E$.

By Lemma 2.4, $F^{\prime \prime}(E)$ is soluble. Hence, $F^{\prime \prime}(E)=F(E)$ by Lemma 2.6. If $F^{\prime \prime}(E)=E$, then by Lemma 2.5 , we conclude that $G$ is supersoluble, a contradiction. Therefore, $F^{*}(E)$ is a proper subgroup of $E$.

(2) Let $p$ be the smallest prime dividing the order of $F$ and $P$ be a Sylow $p$-subgroup of $F$. Then $p>2$.

Assume that $p=2$. Write $V / P=F^{*}(E / P)$. Let $Q$ be a Sylow $q$-subgroup of $E$, where $q \neq 2$. Then $P Q$ is 2-nilpotent by the hypothesis and Lemma 2.4. Since $P$ is normal in G, $P Q$ is nilpotent. It is immediate that $O^{2}(E) \leq C_{E}(P)$. Consider the normal subgroup $W=O^{2}(V) P$ of $E$. Then $W / P$ is a quasinilpotent group by Lemma 2.6. We claim that every chief factor of $W$ below $P$ is central in $W$. In fact, let $H / K$ be a chief factor of $W$ with $H \leq P$ and $W_{p} / P$ a Sylow $p$-subgroup of $W / P$. Then $W_{p}$ is a Sylow $p$-subgroup of $W$ and $H / K \cap Z\left(W_{p} / K\right) \neq 1$. Let $L / K$ be a subgroup of order $p$ contained in $H / K \cap Z\left(W_{p} / K\right)$. Then $L / K$ is centralized by $W_{p} / K$. Besides, since $O^{2}(E) \leq C_{E}(P), L / K$ is centralized by $W_{q} K / K$ for every Sylow $q$ subgroup $W_{q}$ of $W$ with $q \neq p$. Hence, $L / K$ is centralized by $W / K$. It follows that $H / K=$ $L / K$ is a central chief factor of $W$, as desired. Therefore $W$ is a quasinilpotent group and $W \leq F^{*}(E)=F(E)$. Thus, $W$ is nilpotent and $V / F(E)$ is a 2-group, which shows that $V$ is soluble. Hence, $V / P=F^{*}(E / P)$ is nilpotent. Let $R$ be a Sylow 2-subgroup of $V$. Then $R$ is 
normal in $V$. Let $H$ be a Hall $2^{\prime}$-subgroup of $V$. Then $H$ stabilizes the series $R \geq P \geq 1$ and so $H \leq C_{V}(R)$ (see [4, Lemma 3.2.3] or [8, Ch.5, Theorem 3.2]). Now we have that $V$ is nilpotent and so $V=F$. By Lemma 2.1, G/P satisfies the hypothesis. The choice of $G$ implies that $G / P$ is supersoluble and so $G$ is supersoluble by Lemma 2.5 , a contradiction. Hence, $p$ is an odd prime.

(3) If for some normal subgroup $D$ of $G$ we have $D \leq P \cap Z_{\infty}^{\mathcal{U}}(G), 1=D_{0} \leq D_{1} \leq \cdots \leq$ $D_{t}=D$ is a chief series of $G$ below $D$ and $C=C_{1} \cap C_{2} \cap \cdots \cap C_{t}$, where $C_{i}=C_{G}\left(D_{i} / D_{i-1}\right)$, then $E \leq C$.

Since $D \leq Z_{\infty}^{\mathcal{U}}(G), G / C_{G}\left(D_{i} / D_{i-1}\right)$ is cyclic and so $G / C$ is Abelian. Thus, $G /(E \cap C)$ is supersoluble by the hypothesis. By (1) $F^{\prime \prime}(E)=F(E) \leq E \cap C$, from which we conclude that $F^{*}(E \cap C)=F^{*}(E)$ by Lemma 2.6. Hence, the hypothesis is still true for $(G, E \cap C)$. The minimality of $|G|+|E|$ implies that $E \leq C$.

(4) $P$ is not cyclic.

If not, then $E$ stabilizes a chain of subgroups of $P$ by (3). It follows from [4, Lemma 3.2.3] that $E / C_{E}(P)$ is a $p$-group. Thus, $O^{p}(E) \leq C_{E}(P)$. Similar to (2), we have that the pair $(G / P, E / P)$ satisfies the hypothesis. Therefore, the minimal choice of $(G, E)$ implies that $G / P$ is supersoluble. Hence, $G$ is supersoluble by Lemma 2.5 , a contradiction.

Final contradiction.

By (4) and [8, Ch.5, Theorem 3.13], $P$ possesses a characteristic subgroup $D$ of exponent $p$ such that every non-trivial $p^{\prime}$-automorphism of $P$ induces a non-trivial automorphism of $D$. Then $D \leq Z_{\infty}^{\mathcal{U}}(G)$ by Lemma 3.1. Let $1=D_{0} \leq D_{1} \leq \cdots \leq D_{t}=D$ be a chief series of $G$ below $D$ and $C=C_{1} \cap C_{2} \cap \cdots \cap C_{t}$ where $C_{i}=C_{G}\left(D_{i} / D_{i-1}\right)$. Then $E \leq C$ via (3). Thus, $E / C_{E}(D)$ is a $p$-group, which implies that $E / C_{E}(P)$ is also a $p$-group. Equivalently, we have $O^{p}(E) \leq C_{E}(P)$. Using an analogous argument as in (2), we have that $G / P$ is supersoluble and consequently $G$ is supersoluble by Lemma 2.5 , which violates the choice of $G$.

\section{Some applications}

Since many relevant families of subgroups, such as normal subgroups, $(S$-)quasinormal subgroups (or $(S$-)permutable subgroups), $c$-normal subgroups, complemented subgroups, $c$-supplemented subgroups, weakly $S$-supplemented subgroups and $S$-quasinormally embedded subgroups, enjoy the $\lambda$-supplementary property, a lot of nice results follow from Theorem 3.2.

Recall first some concepts of subgroups mentioned above. Let $H$ be a subgroup of a group $G$. We call $H$ a complemented subgroup of $G$ if there exists a subgroup $T$ of $G$ such that $G=H T$ and the intersection of $H$ and $T$ is trivial. $H$ is said to be $c$-normal ( $c$ supplemented) in $G$ if $G$ has a normal subgroup (a subgroup) $T$ such that $G=H T$ and $H \cap T \leq H_{G}$, where $H_{G}$ denotes the largest normal subgroup of $G$ contained in $H$ (see [9-11]).

Now, we give three corollaries which follow directly from Theorem 3.2.

Corollary 4.1 [12] If every minimal subgroup of a group $G$ of odd order is normal in G, then $G$ is supersoluble.

Corollary 4.2 [13] Let $G$ be a group with a normal soluble subgroup $E$ such that G/E is supersoluble. If all subgroups of $F(E)$ of prime order or order 4 are c-normal in $G$, then $G$ is supersoluble. 
Corollary 4.3 [14] Let $G$ be a soluble group and E be a normal subgroup of $G$ such that $G / E$ is supersoluble. If every minimal subgroup of $F(E)$ is complemented in $G$, then $G$ is supersoluble.

The following result can be established by applying a similar argument as that in the proof of Theorem 3.2.

Theorem 4.4 Let $\mathcal{F}$ be a saturated formation containing all supersoluble groups. A group $G \in \mathcal{F}$ if and only if there exists a normal subgroup $E$ of $G$ such that $G / E \in \mathcal{F}$ and for every non-cyclic Sylow subgroup $P$ of $F^{*}(E)$, every cyclic subgroup $H$ of $P$ of prime order or order 4 (if $P$ is a non-Abelian 2-group and $H \nsubseteq Z_{\infty}(G)$ ) is $\lambda$-supplemented in $G$ provided that $H$ does not possess any supersoluble supplement in $G$.

By Theorem 4.4, we have

Corollary 4.5 [15] Let $\mathcal{F}$ be a saturated formation containing all supersoluble groups and $G$ be a group with a normal soluble subgroup $E$ such that $G / E \in \mathcal{F}$. If all minimal subgroups and all cyclic subgroups with order 4 of $F(E)$ are c-normal in $G$, then $G \in \mathcal{F}$.

Corollary 4.6 [16] Let $\mathcal{F}$ be a saturated formation containing all supersoluble groups and $G$ be a group with a normal subgroup $E$ such that $G / E \in \mathcal{F}$. If all minimal subgroups and all cyclic subgroups with order 4 of $F^{*}(E)$ are c-normal in $G$, then $G \in \mathcal{F}$.

Corollary 4.7 [17] Let $\mathcal{F}$ be a saturated formation containing all supersoluble groups and $G$ be a group with a normal subgroup $E$ such that $G / E \in \mathcal{F}$. If every minimal subgroup and each cyclic subgroup with order 4 of $F^{*}(E)$ is c-supplemented in $G$, then $G \in \mathcal{F}$.

Corollary 4.8 [18] Let $\mathcal{F}$ be a saturated formation containing all supersoluble groups and $G$ be a group with a normal soluble subgroup $E$ such that $G / E \in \mathcal{F}$. If all minimal subgroups of $F\left(E \cap G^{\prime}\right)$ are complemented in $G$, then $G \in \mathcal{F}$.

Corollary 4.9 [19] Let $\mathcal{F}$ be a saturated formation containing all supersoluble groups and $G$ be a group with a normal soluble subgroup $E$ such that $G / E \in \mathcal{F}$. If all minimal subgroups and all cyclic subgroups with order 4 of $F(E)$ are $S$-permutable in $G$, then $G \in \mathcal{F}$.

Corollary 4.10 [20] Let $\mathcal{F}$ be a saturated formation containing all supersoluble groups and $G$ be a group with a normal subgroup $E$ such that $G / E \in \mathcal{F}$. If every cyclic subgroup of $F^{*}(E)$ of prime order or order 4 is $S$-permutable in $G$, then $G \in \mathcal{F}$.

Corollary 4.11 [21] Let $\mathcal{F}$ be a saturated formation containing all supersoluble groups and $G$ be a group with a normal subgroup $E$ such that $G / E \in \mathcal{F}$. If each minimal subgroup of $F^{*}(E)$ of order prime or 4 is $S$-quasinormally embedded in $G$, then $G \in \mathcal{F}$.

In [2], Skiba proposed the following question.

Question 4.12 Let $\mathcal{F}$ be a saturated formation containing all supersoluble groups and $G$ be a group with a normal subgroup $E$ such that $G / E \in \mathcal{F}$. Suppose that every non-cyclic Sylow subgroup $P$ of $F^{*}(E)$ has a subgroup $D$ such that $1<|D|<|P|$ and all subgroups 
$H$ of $P$ with order $|H|=|D|$ and with order $2|D|$ (if $P$ is a non-Abelian 2-subgroup and $|P: D|>2)$ are weakly $s$-supplemented in $G$. Is then $G \in \mathcal{F}$ ?

Our last corollary gives a positive answer to the above question in the case of minimal subgroups.

Corollary 4.13 Let $\mathcal{F}$ be a saturated formation containing all supersoluble groups and $G$ be a group with a normal subgroup $E$ such that $G / E \in \mathcal{F}$. If each cyclic subgroup of $F^{*}(E)$ of order prime or 4 is weakly $S$-supplemented in $G$, then $G \in \mathcal{F}$.

\section{Competing interests}

The authors declare that they have no competing interests.

\section{Acknowledgements}

The author would like to express his sincere thanks to the referees whose careful reading and important comments on this article led to a number of improvements. This work was supported by the Scientific Research Foundation of Chongqing University of Arts and Sciences (Grant Nos. R2012SC21, Z2012SC25) and the National Natural Science Foundation of China (Grant Nos. 11271301, 11171364, 11001226).

Received: 11 September 2012 Accepted: 30 October 2012 Published: 15 November 2012

\section{References}

1. Ballester-Bolinches, A, Pedraza-Aguilera, MC: Sufficient conditions for supersolubility of finite groups. J. Pure Appl. Algebra 127, 113-118 (1998)

2. Skiba, AN: On weakly s-permutable subgroups of finite groups. J. Algebra 315, 192-209 (2007)

3. Li, JB, Chen, GY, Yan, YX: New characterizations of $p$-supersolubility of finite groups. Commun. Algebra $40(12)$, 4372-4388 (2012)

4. Guo, WB: The Theory of Classes of Groups. Kluwer Academic, Dordrecht (2000)

5. Asaad, M: Finite groups with certain subgroups of Sylow subgroups complemented. J. Algebra 323, 1958-1965 (2010)

6. $L i, J B, Y u, D P, C h e n, S M:$ On $\lambda$-supplemented minimal subgroups of finite groups. J. Chongqing Normal University: Natural Science Edition (to appear)

7. Huppert, B, Blackburn, N: Finite Groups III. Springer, Berlin (1982)

8. Gorenstein, D: Finite Groups. Chelsea Publishing Company, New York (1980)

9. Ballester-Bolinches, A, Wang, YM, Guo, X: c-Supplemented subgroups of finite groups. Glasg. Math. J. 42, 383-389 (2000)

10. Wang, YM: c-Normality of groups and its properties. J. Algebra 180(3), 954-965 (1996)

11. Wang, YM: Finite groups with some subgroups of Sylow subgroups c-supplemented. J. Algebra 224, 467-478 (2000)

12. Buckley, J: Finite groups whose minimal subgroups are normal. Math. Z. 116, $15-17$ (1970)

13. Li, D, Guo, X: The influence of $c$-normality of subgroups on the structure of finite groups, II. Commun. Algebra 26, 1913-1922 (1998)

14. Li, D, Guo, X: On complemented subgroups of finite groups. Chin. Ann. Math. 22B(2), 249-254 (2001)

15. Wei, HQ: On c-normal maximal and minimal subgroups of Sylow subgroups of finite groups. Commun. Algebra 29 2193-2200 (2001)

16. Wei, HQ, Wang, YM, Li, YM: On c-normal maximal and minimal subgroups of Sylow subgroups of finite groups, II. Commun. Algebra 31, 4807-4816 (2003)

17. Wei, HQ, Wang, YM, Li, YM: On c-supplemented maximal and minimal subgroups of Sylow subgroups of finite groups. Proc. Am. Math. Soc. 32(8), 2197-2204 (2004)

18. Guo, X, Shum, KP: Complementarity of subgroups and the structure of finite groups. Algebra Collog. 13(1), 9-16 (2006)

19. Asaad, M, Csörgö, P: Influence of minimal subgroups on the structure of finite groups. Arch. Math. 72, 401-404 (1999)

20. Li, YM, Wang, YM: The influence of minimal subgroups on the structure of a finite group. Proc. Am. Math. Soc. 131, 245-252 (2002)

21. Li, YM, Wang, YM: On $\pi$-quasinormally embedded subgroups of finite groups. J. Algebra 281, 109-123 (2004)

doi:10.1186/1029-242X-2012-266

Cite this article as: Li: A characterization of supersolubility of finite groups. Journal of Inequalities and Applications 2012 2012:266 